\title{
Meditative Movements for Patients with Type 2 Diabetes: A Systematic Review and Meta-Analysis
}

\author{
Tingwei Xia, ${ }^{1}$ Yue Yang, ${ }^{2}$ Weihong Li ${ }^{1},{ }^{1}$ Zhaohui- Tang, ${ }^{1}$ Qingsong Huang, ${ }^{1}$ Zongrun Li, ${ }^{1}$ \\ and Yongsong Guo ${ }^{1}$ \\ ${ }^{1}$ Chengdu University of TCM, Chengdu, Sichuan Province, China \\ ${ }^{2}$ Department of TCM, Qingyang District People's Hospital, Chengdu, Sichuan Province, China
}

Correspondence should be addressed to Weihong Li; lwh@cdutcm.edu.cn

Received 20 August 2019; Accepted 28 December 2019; Published 1 February 2020

Academic Editor: Martin Offenbaecher

Copyright (C) 2020 Tingwei Xia et al. This is an open access article distributed under the Creative Commons Attribution License, which permits unrestricted use, distribution, and reproduction in any medium, provided the original work is properly cited.

Objective. Physical activity plays a specific role in the fundamental aspect of diabetes care. It is necessary to develop exercise programs for these patients. The aim of this systematic review is to summarize current evidence regarding the effectiveness of meditative movement in patients with type 2 diabetes. Methods. The following databases were searched: PubMed, CENTRAL, Web of Science, Ovid LWW, and EMBASE. Two independent investigators searched and screened the studies by finding duplications, excluding irrelevant titles and abstracts, and then selecting eligible studies by reviewing full texts. 21 studies fulfilled the inclusion criteria. Meta-analyses were performed on glycated hemoglobin (HbAlc), fasting blood glucose (FBG) and postprandial blood glucose (PPBG), total cholesterol (TC), triglycerides (TG), high-density lipoprotein cholesterol (HDL-C), lowdensity lipoprotein cholesterol (LDL-C), and body mass index (BMI). Results. Meta-analyses showed that meditative movements significantly improved FBG, HbA1c, PPBG, TC, LDL-C, and HDL-C. No improvement was found in BMI. Conclusions. The results demonstrated a favorable effect or tendency of meditative movements to improve blood glucose and blood lipid levels in patients with type 2 diabetes mellitus. The special effects of meditative movements in type 2 diabetes mellitus patients need further research.

\section{Background}

Physical activity is an important part of the diabetes lifestyle management and negatively associated with the risk of type 2 diabetes mellitus (T2DM). It plays a specific role in the fundamental aspect of diabetes care [1-3]. Type 2 diabetes is one of the most common diseases in older adults. However, the incidence of children, adolescents, or young people is on the rise, due to the rising level of obesity, lack of physical activity, and poor diet [2]. As the International Diabetes Federation reported, there are approximately 451 million people (ages 18-99 years) with diabetes in the world [3]. And approximately $90-95 \%$ of all cases are type 2 diabetes [2]. By 2017, nearly 5 million people between the ages of 20 and 99 had died of diabetes and its complications [4]. At the same time, there are 374 million people with impaired glucose tolerance who are at high risk of developing diabetes [4].
Diabetic complications affect hundreds of millions of patients with type 2 diabetes [5]. T2DM patients have a high risk of liver fibrosis and liver steatosis $[6,7]$. Due to the presentation and progression of these complications, patients may lose their vision, kidney, and nerve function. Their activity and cognitive ability may be impaired, and their quality of life may deteriorate. This leads to limited employment and productivity and increased costs for the patient and society [8-11].

Meditative movements, combining breath control, relaxation, musculoskeletal stretching, and a meditative state of mind, have been shown to be effective for treating type 2 diabetes [12]. Meditative movements, including Tai Chi, Yoga, and Qigong, reported by the National Health Interview Survey, are popular among American adults in workplace [13]. Yoga and Tai Chi, especially, are recommended by the American Diabetes Association for 
older adults with type 2 diabetes to increase flexibility, muscular strength, and balance [1]. Plenty of clinical researches have focused on the effectiveness of meditative movements on type 2 diabetes. Present systematic reviews or meta-analyses about meditative movements have shown that it is beneficial to chronic obstructive pulmonary disease, sleep quality, cancer, and major depressive disorder [14-17].

However, the systematic review and meta-analysis of meditative movements on type 2 diabetes have not been conducted. Therefore, we performed a systematic review and meta-analysis to evaluate the effectiveness of meditative movements as a complementary therapy for patients with type 2 diabetes.

\section{Data and Methods}

This review was performed according to our previous protocol [18]. Our protocol of this systematic review and metaanalysis on PROSPERO was registered in advance (no. CRD42019128495, https://www.crd.york.ac.uk/PROSPERO).

2.1. Data Sources and Search Strategies. The following databases were searched using the developed search strategy [18] from inception to December 2018: PubMed, Cochrane Central Register of Controlled Trials (CENTRAL), Web of Science, Ovid LWW, and EMBASE.

2.2. Inclusion and Exclusion Criteria. We identified studies using the following inclusion criteria as in our protocol [18]: participants (with a clear diagnosis of type 2 diabetes), intervention (Tai Chi or Qigong or Yoga), control (any type of control group), primary outcomes (HbA1c, FBG, and PPBG), secondary outcomes (TC, TG, HDL-C, LDL-C, and $\mathrm{BMI}$ ), and study type (randomized controlled trials (RCTs)).

2.3. Trials Inclusion and Data Extraction. Two investigators independently searched and screened the studies. The process of study selection was performed using the methods according to the PRISMA guidelines [19]. Data extraction was performed by two investigators independently. Data extraction contained, in addition to outcomes, information regarding country of origin, number of randomized participants, number of participants included in type of intervention, frequency of intervention, and duration of intervention. Finally, all differences were resolved by consensus.

2.4. Trials Quality Assessment. Definitions in the assessment of bias risk of a trial were conducted according to the Cochrane Handbook criteria for judging the ROB with the "risk of bias" assessment tool [20]. The following domains should be evaluated: random sequence generation, allocation concealment, blinding of participants and investigators, the blindness of outcome assessments, incomplete outcome data, selective outcome reporting, and other biases. The quality of studies was divided into three categories: low, unclear, or high bias.

2.5. Statistical Analysis. We used the RevMan 5.3.0 provided by Cochrane Collaboration to analyze the results of the studies. This meta-analysis only included continuous data, so we expressed them as the mean \pm standard deviation and then calculated the standardized mean difference (SMD) and obtained the two-sided $P$ value and 95\% confidence interval (CI). The complete case data was used as the analysis data. The degree of heterogeneity was quantified using the $\chi^{2}$ test and $I^{2}$ value. We performed subgroup analysis according to total sample size $(>60$ versus $\leq 60)$, duration ( $>3$ months versus $\leq 3$ months), control type (nonexercise versus other active exercises), type of meditative movement (Tai Chi/Qigong versus Yoga), and region (Asia versus non-Asia), as well as a sensitivity analysis if necessary. A test for the interaction between the treatment and subgroups was performed to examine whether treatment effects differed among subgroups. An interaction of $P$ value $\geq 0.05$ was considered to indicate that the effect of treatment did not differ significantly among subgroups. Publication bias was assessed by visual inspection of a funnel plot.

\section{Results}

3.1. Literature Screening. We retrieved 818 original papers from the electronic bibliographic databases. The full text of 127 articles was assessed according to the predetermined inclusion criteria. Finally, 21 studies fulfilled the inclusion criteria and were further analyzed [21-44]. The detailed process of the studies evaluation and the reasons for exclusion are shown in Figure 1.

3.2. Characteristics of Included Studies. The characteristics of these included trials are described in Table 1. Among them, the data of three studies were reported by six different articles [24-27, 37, 38]. Patients included in these studies were from China [23, 39], Taiwan (China) [29], India [22, 24-27, 31-35], Iran [41], Japan [42], Thailand [30], Australia $[40,43,44]$, Cuba $[37,38]$, and USA $[21,28,36]$. Six studies offered Tai Chi [23, 29, 30, 39, 40, 44], three studies offered Qigong [28, 42, 43], and twelve studies offered Yoga $[21,22,24-27,31-38,41]$. The sample sizes of the included studies ranged from 10 to 277 . The treatment duration lasted from 45 days to 36 weeks. The frequency ranged from 2 to 7 times weekly, and exercise time lasted 10-120 min per session. Controls were divided into nonexercise groups and other active exercise groups. The exercise forms of other active exercise groups include seated calisthenics, stretching, aerobic exercise plus home-based exercise, progressive resistance training, and physical activity. In three studies, two control groups were set up in each, including nonexercise and other active exercises $[28,31,37,38]$. 


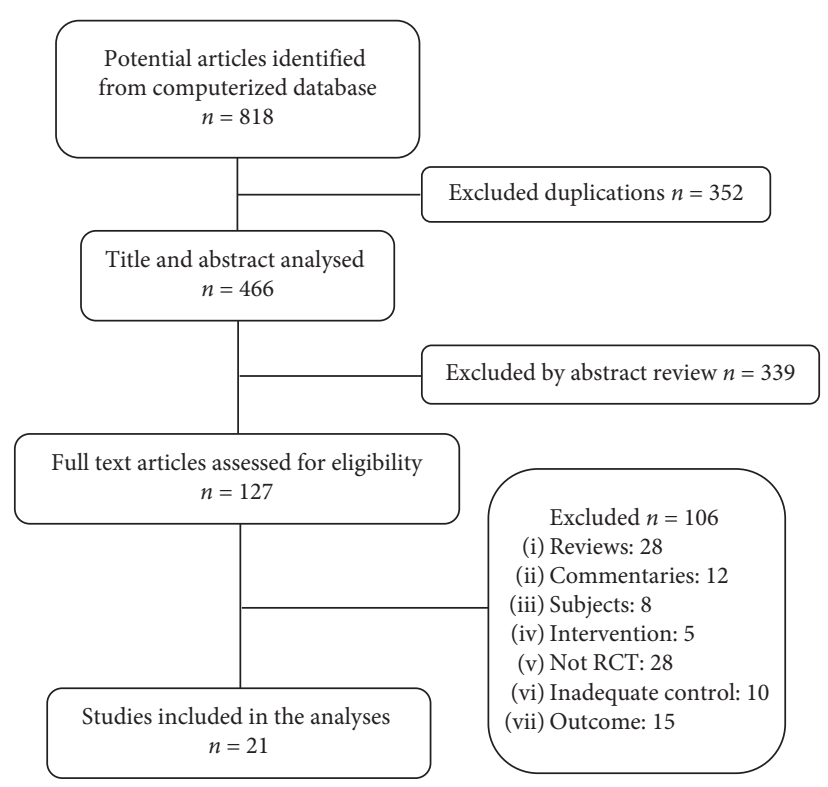

Figure 1: Flow chart of selection process.

3.3. Risk of Bias of Studies. The bias condition of selected studies was shown in Figures 2 and 3. We assessed the risk of bias in all included articles. Eleven studies used the generation of the allocation sequence. Allocation concealment was used in 6 studies. None of the studies blinded their participants. However, nine studies were blinding of outcome assessors to the treatment allocation, whereas the risk of selective reporting bias was not reported in most studies.

\section{Outcome}

4.1. Glycemic Control. Sixteen RCTs, with three studies setting up two control groups in each, reported FBG as a primary outcome. We split the study with two control groups into two sets of data for summary analysis. A total of 19 sets of data were included. The combined result was statistically significant $(\mathrm{SMD}=0.81,95 \%$ CI $(0.38,1.24)$, $P=0.0002$ ) compared to the control group, with high heterogeneity $\left(I^{2}=93 \%, P<0.00001\right.$ ) (Figure 4$)$. We carried out sensitivity analyses to explore potential sources of heterogeneity, and the results did not change substantively. The heterogeneity ranged from $67 \%$ to $93 \%$. So, we conducted subgroup analyses and interaction tests according to the total sample size, duration, control type, intervention type, and region. Test for interaction showed significant results between subgroups of the nonexercise and other active exercises $(P$-interaction $=0.002)$. The result indicated that the difference of the control types was partly the reason why there was severe heterogeneity in the overall analysis. The detailed results are shown in Table 2.

Thirteen studies reported HbAlc. Sixteen sets of data were included. The heterogeneity was high $(P<0.00001$, $\left.I^{2}=92 \%\right)$. We carried out sensitivity analyses to investigate the potential sources of heterogeneity. After removing one set of data, the results changed obviously. The heterogeneity was calculated as $P=0.64, I^{2}=0 \%$. The combined result was statistically significant $(\mathrm{SMD}=0.36,95 \% \mathrm{CI}(0.24,0.48)$, and $P<0.00001$ ) (Figure 5). It showed that one study was the potential source of heterogeneity. However, when we looked up the study again, we did not find differences in methodology and other aspects. The study showed that meditation movements had more significant effects on $\mathrm{HbAlc}$ than other studies. Five studies reported the PPBG. It showed a favorable effect of meditation movements on reducing PPBG $(\mathrm{SMD}=0.30,95 \% \mathrm{CI}(0.14,0.46)$, and $P=0.0002)$, with low heterogeneity $\left(P=0.29, I^{2}=19 \%\right)$ (Figure 6).

4.2. Lipid Profile. The aggregated results suggested that the meditation movements had significant effects on TC $(\mathrm{SMD}=0.64,95 \% \mathrm{CI}(0.02,1.26)$, and $P=0.04 ; P$ for heterogeneity $<0.00001, \quad \mathrm{I}^{2}=95 \%$ ) (Figure 7 ), LDL-C $(\mathrm{SMD}=0.61,95 \% \mathrm{CI}(0.16,1.06)$, and $P=0.008 ; P$ for heterogeneity $<0.00001, I^{2}=88 \%$ ) (Figure 8 ), triglycerides $(\mathrm{SMD}=0.19,95 \% \mathrm{CI}(0.06,0.31)$, and $P=0.004 ; \mathrm{P}$ for heterogeneity $=0.14, I^{2}=33 \%$ ) (Figure 9), and HDL-C $(\mathrm{SMD}=-0.53,95 \% \mathrm{CI}(-0.90,-0.15)$, and $P=0.006 ; P$ for heterogeneity $<0.00001, \mathrm{I}^{2}=85 \%$ ) (Figure 10).

It showed no effects of meditation movements on reducing BMI (SMD $=0.42,95 \%$ CI $(-0.20,1.03)$, and $P=0.18)$ with low heterogeneity $\left(P<0.00001, I^{2}=95 \%\right)$ (Figure 11).

4.3. Publication Bias. The FBG included in the study was selected as an indicator. It can be seen that the graph is not obviously asymmetrical (Figure 12). There might have been no publication bias in the comparison of meditation movements and the control group.

\section{Discussion}

Meditative movements (specifically Tai Chi, Qigong, and Yoga), including a focus of the mind on the body and 


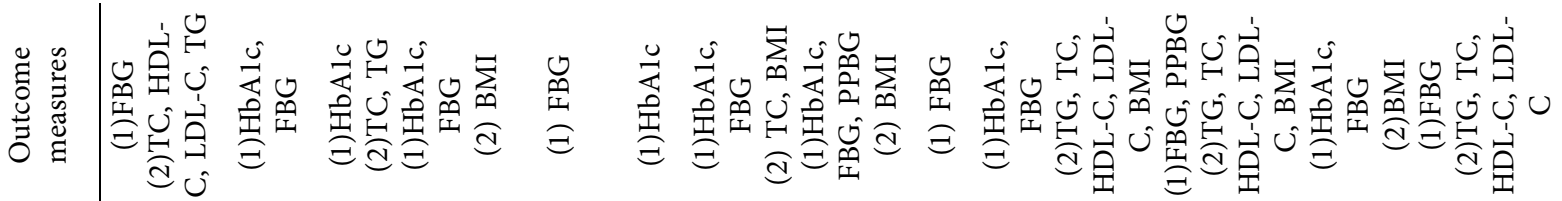

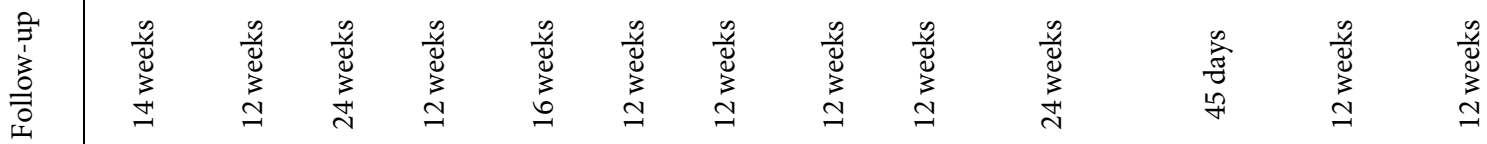

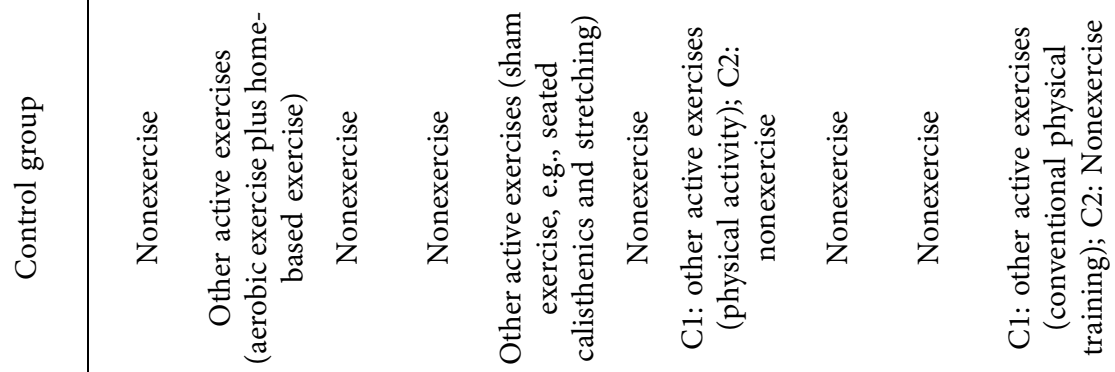

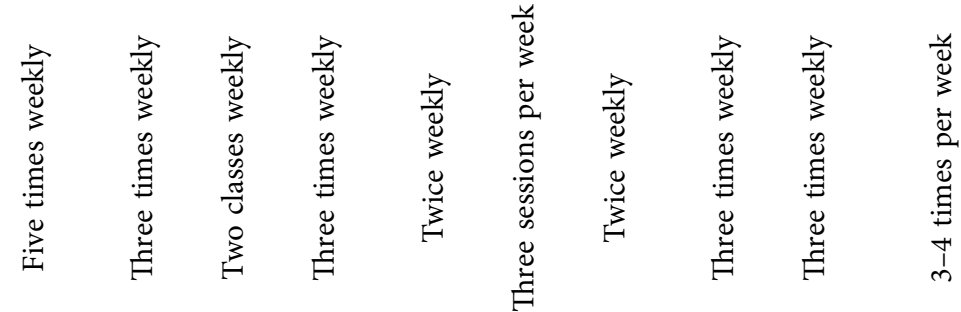

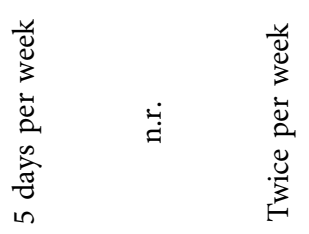

竞

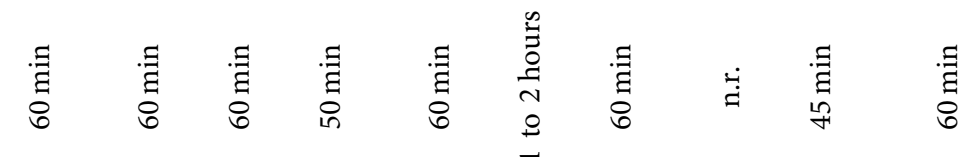

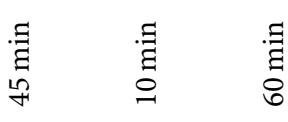

岁落

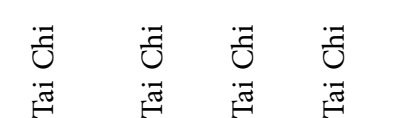

$\begin{array}{ll}\text { ปี } & \text { ปี } \\ \text { : } & \text { 표 }\end{array}$

$\stackrel{\square}{\circ}$

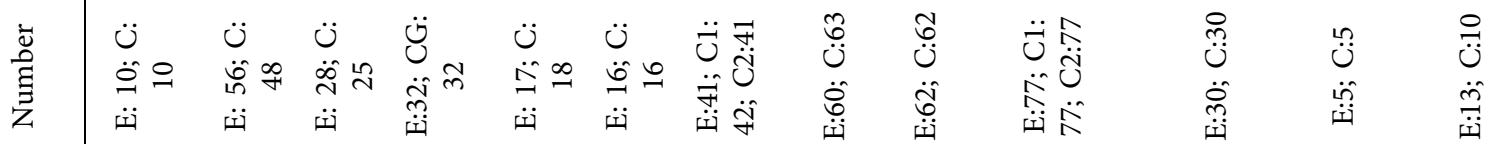

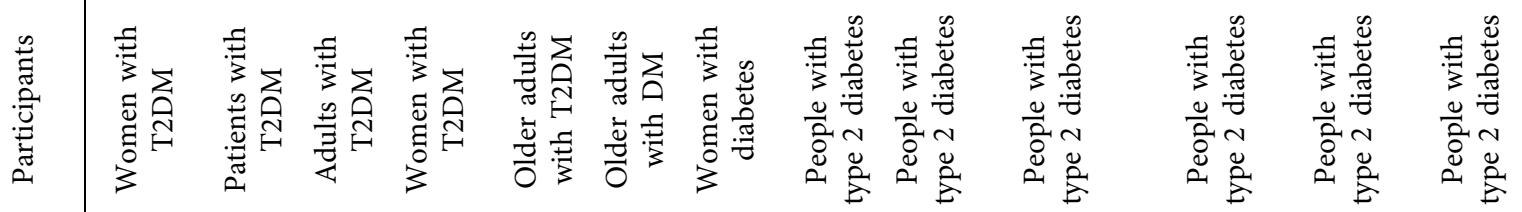

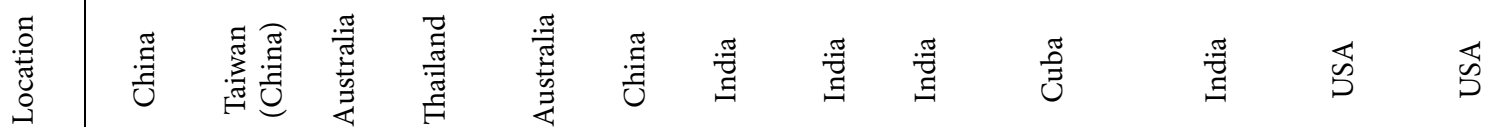

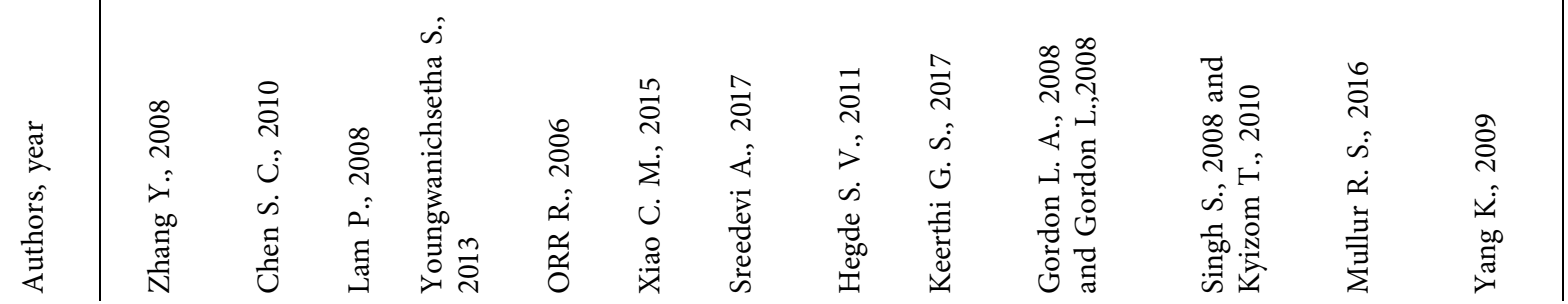




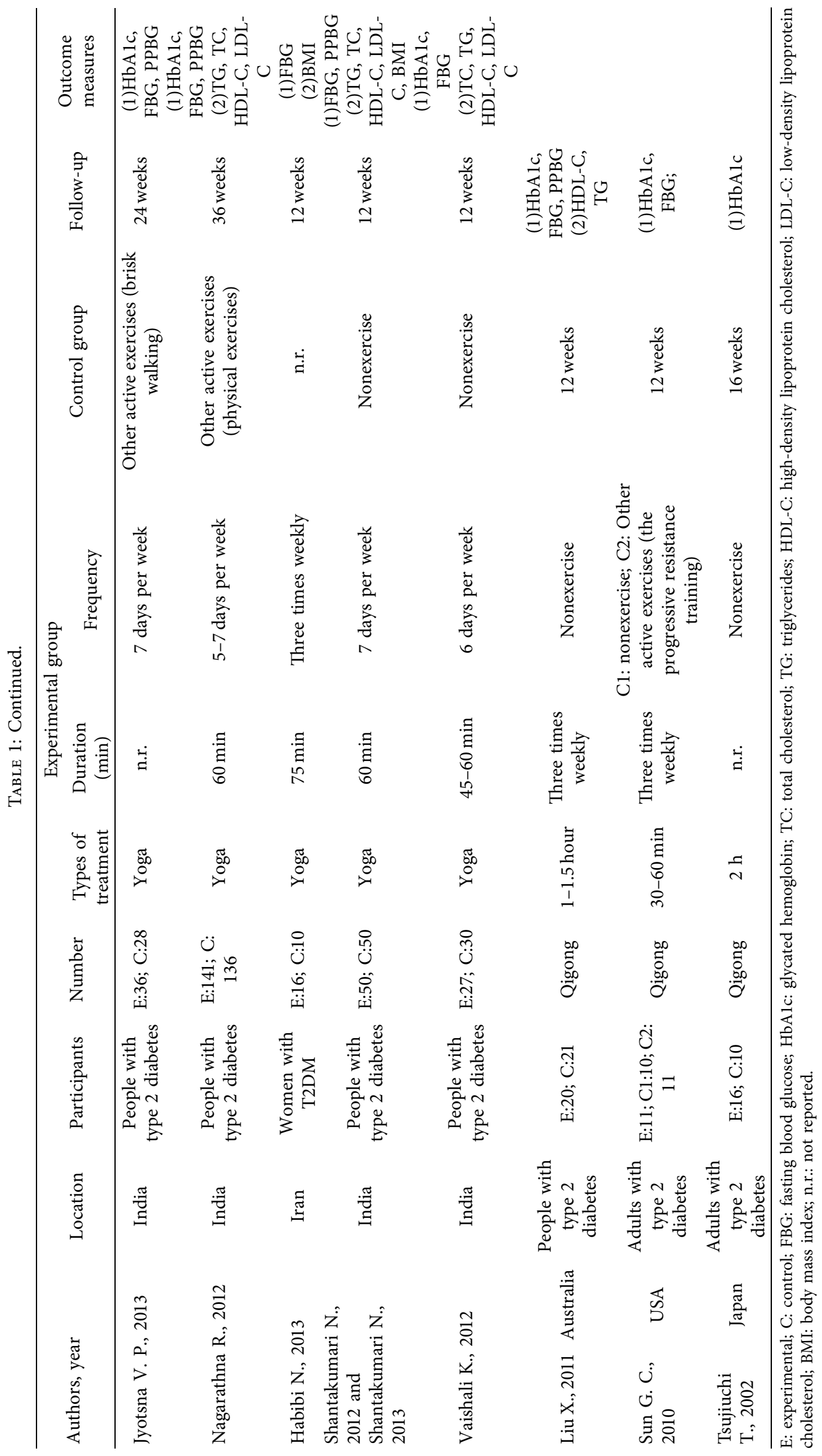




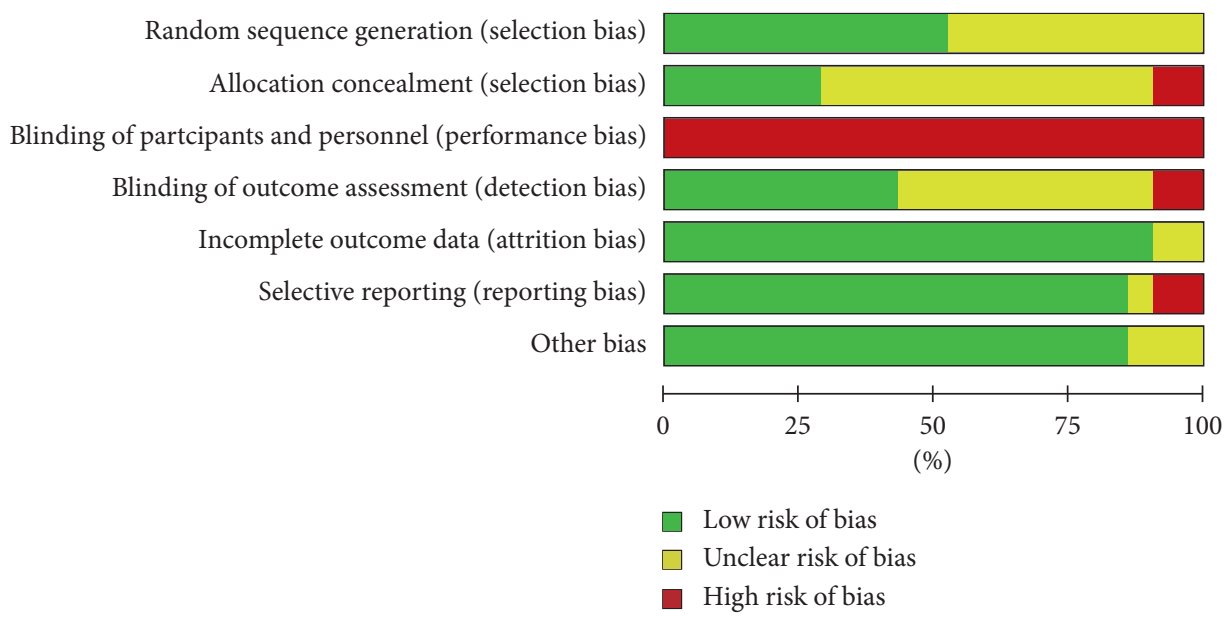

Figure 2: Risk of bias graph.

breathing for deep relaxation, are special forms of exercise. More and more studies have been conducted on the effectiveness of these practices in health and healing [14]. As the first systematic review and meta-analysis synthesize the evidence of the effects of meditative movements on type 2 diabetes, we found that meditative movements may have positive effects on the treatment of type 2 diabetes. This evidence suggests that there is a possibility for using these exercises as an augmentation approach to control blood glucose for type 2 diabetes.

5.1. Summary of Main Results. The present results showed that the meditative movements significantly improved FBG, HbA1c, PPBG, TC, LDL-C, and HDL-C in patients with type 2 diabetes mellitus. No improvement was found in BMI.

As for the primary outcomes, significant heterogeneity was noted during our analyses of FBG and HbA1c. Sensitivity analyses were carried out to explore the potential sources of heterogeneity for FBG. We found that the heterogeneity or the synthesized results of studies on FBG did not change substantively. Therefore, subgroup analyses and interaction tests were carried out to investigate the impact of various exclusion criteria according to the total sample size, duration, control type, intervention type, and region. No evidence of heterogeneity was observed within the total sample size, duration, intervention type, and region. However, the overall combined effects of the trials showed significant results between subgroups of the nonexercise and other active exercises. It indicated that the reason for heterogeneity might be caused by the difference of the control types. Although the results showed a significant difference in reducing FBG between meditative movements and other active exercises, it was more significant compared to the nonexercise group. There is no doubt that other active exercises had a better effect on lowering blood sugar than nonexercise. Sensitivity subgroup analyses were also conducted to explore the potential sources of heterogeneity for HbAlc. We found only one study was the potential source of heterogeneity where no differences were found in methodology and other aspects.
It showed more significant effects of meditation movements on HbA1c than other studies.

Psychological stress has been proven to play a role in the etiology of type 2 diabetes [45]. It is regarded both as a predictor of new-onset type 2 diabetes and as a prognostic factor in people with existing type 2 diabetes. The disturbances across multiple biological systems reflecting chronic allostatic load might exist [46]. Numerous studies have shown that it is a common independent risk factor for disease occurrence [47-50]. Meditative movements could be regarded as a combination of mindfulness intervention and physical activity [51]. This characteristic determines that its intervention in type 2 diabetes is multifaceted. Diaphragmatic breathing practice might be beneficial to reduce negative subjective and physiological consequences of stress in healthy adults [52]. This might partly explain why meditative movements have a more positive influence on type 2 diabetes, comparing to other active exercises and nonexercises. Yoga and Tai Chi are mainly recommended to increase flexibility, muscle strength, and balance, which shows that the particularity of meditative movements is not yet well-known. Plenty of studies have shown that meditative movements are effective for glucose control in patients with type 2 diabetes [53-56]. It is necessary to develop exercise programs because the optimal form of exercise and appropriate parameters of exercise in type 2 diabetes patients are not yet clear.

5.2. Limitations. Several limitations have to be mentioned. Heterogeneity among the studies was significant. We conducted sensitivity analyses and subgroup analyses. The control types, other active exercises and nonexercises, might be the main source of heterogeneity. To some extent, they could explain the source of heterogeneity. But the risk of bias and heterogeneity could also be caused by study quality or the exercise intensity. Because participants cannot be blinded to the meditative movements, performance bias could not be ruled out. The distribution of the included studies is also a great concern. Most trials were conducted in Asia or America. No studies were from the 


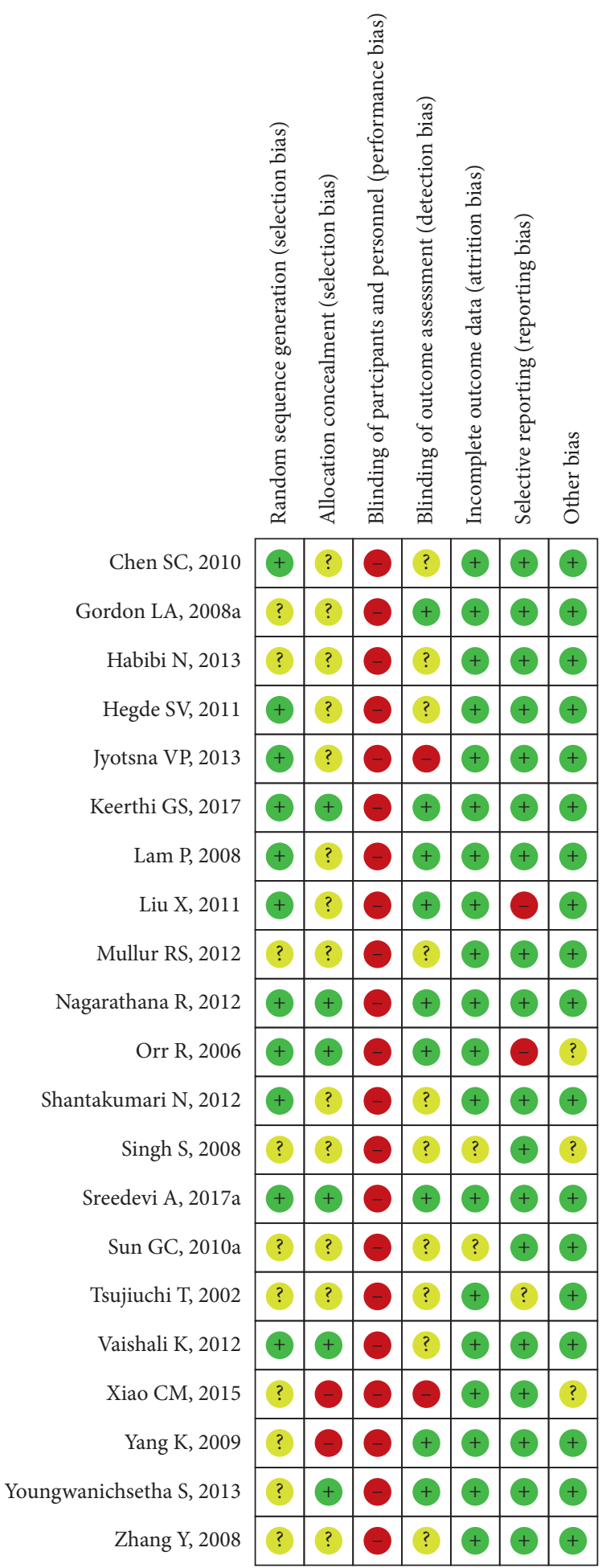

FIGURE 3: Risk of bias summary.

European countries. Due to the limited number of included studies in Qigong, more comprehensive subgroups could not be made. This may have influenced the explanatory effect and the soundness of the pooled effects. Since we only performed a search for English studies, it is possible that articles may have been published in other languages.
5.3. Implications for Research. There are a few points that should be considered in the future. The methodological quality of these studies was poor in random sequence generation, allocation concealment, and blinding of outcome assessment. More studies with rigorous design and normative description are needed in this field. We first summarized the current condition of meditative movements 


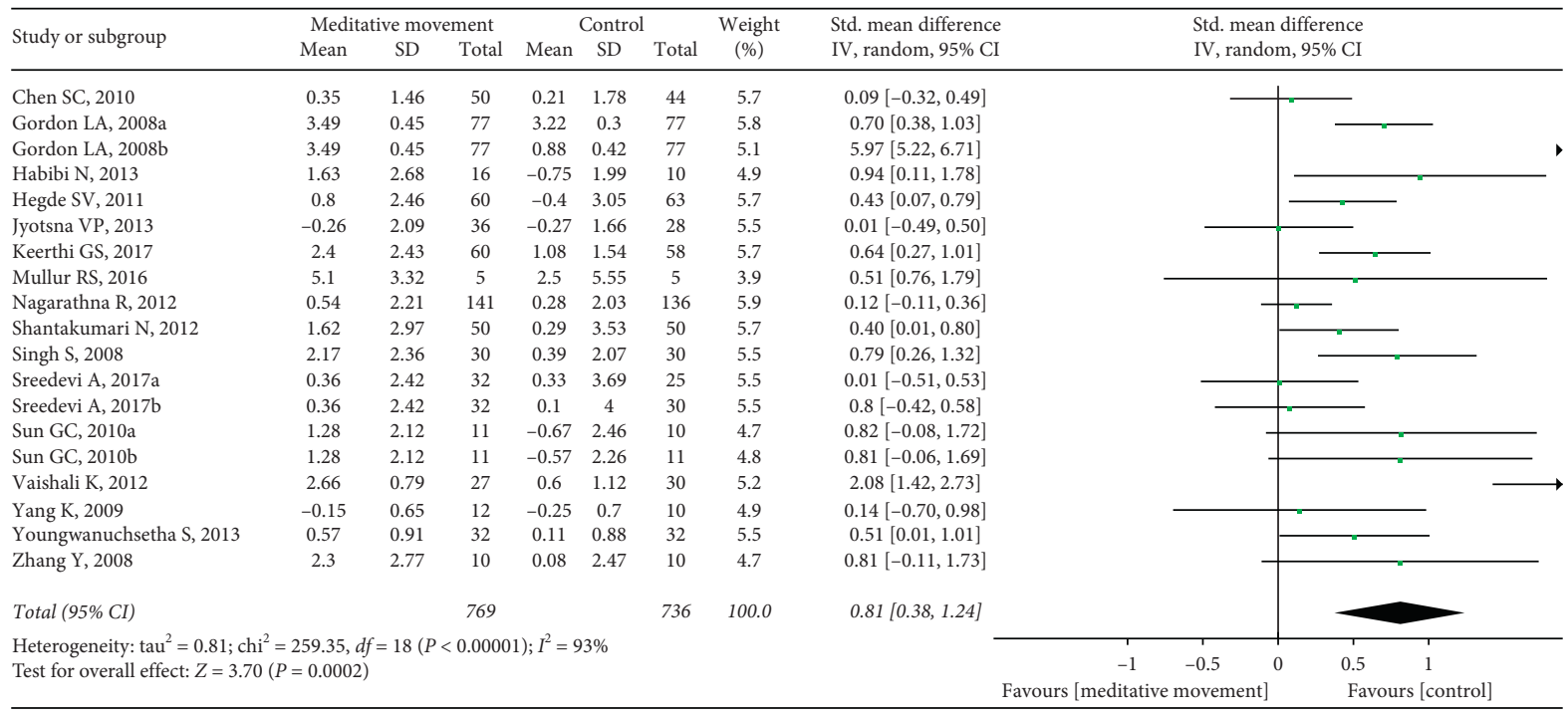

FIGURE 4: Forest plot of the comparison between meditation movements and the control group for the outcome FBG.

TABLE 2: Subgroup analyses based on various exclusion criteria for FBG.

\begin{tabular}{|c|c|c|c|c|c|}
\hline Subgroup & $n$ & SMDs, $\mathrm{mmol} / \mathrm{L}(95 \% \mathrm{CI})$ & $I^{2}(\%)$ & Heterogeneity, $P$ value & $P$ interaction \\
\hline Total sample size & & & & & 0.87 \\
\hline$>60$ & 10 & $0.84(0.22,1.47)$ & 96 & $<0.00001$ & \\
\hline$\leq 60$ & 9 & $0.91(0.38,1.44)$ & 76 & $<0.0001$ & \\
\hline Duration & & & & & 0.11 \\
\hline$>3$ months & 5 & $1.79(0.30,3.28)$ & 98 & $<0.00001$ & \\
\hline$\leq 3$ months & 14 & $0.55(0.29,0.80)$ & 65 & 0.0004 & \\
\hline Control type & & & & & 0.002 \\
\hline Nonexercise & 12 & $1.42(0.71,2.13)$ & 93 & 0.64 & \\
\hline Other active exercises & 6 & $0.27(0.15,0.38)$ & 0 & $<0.00001$ & \\
\hline Intervention type & & & & & 0.71 \\
\hline Tai Chi/Qigong & 5 & $0.74(0.16,1.32)$ & 72 & 0.007 & \\
\hline Yoga & 14 & $0.89(0.35,1.44)$ & 95 & $<0.00001$ & \\
\hline Region & & & & & 0.28 \\
\hline Asia & 13 & $1.50(-0.25,3.25)$ & 78 & $<0.00001$ & \\
\hline Non-Asia & 6 & $0.53(0.25,0.80)$ & 97 & $<0.00001$ & \\
\hline
\end{tabular}

CIs, confidence intervals; n, number of trials; SMDs, standardized mean differences.

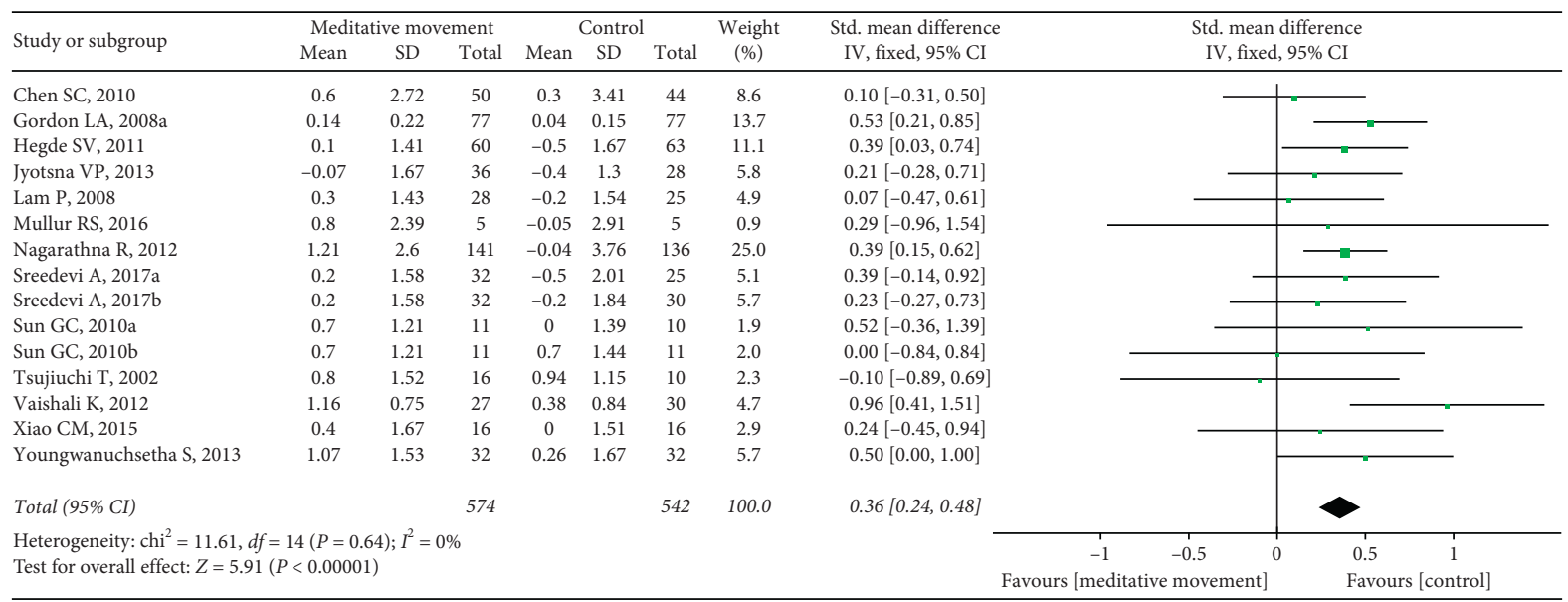

FIGURE 5: Forest plot of the comparison between meditation movements and the control group for the outcome HbA1c. 


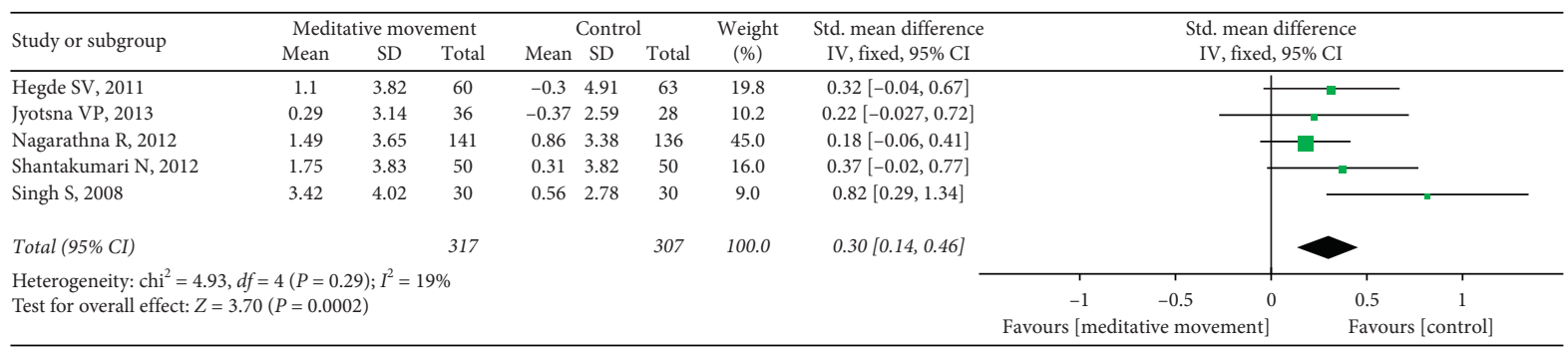

FIGURE 6: Forest plot of the comparison between meditation movements and the control group for the outcome PPBG.

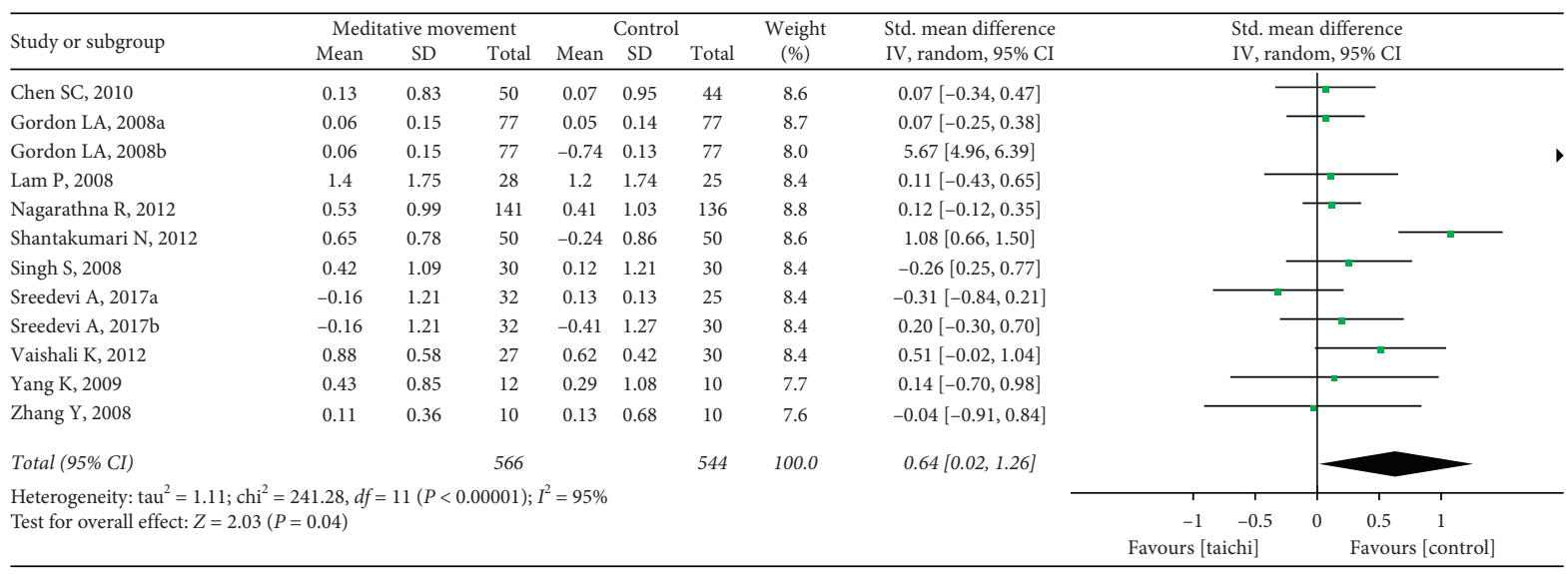

FIgURE 7: Forest plot of the comparison between meditation movements and the control group for the outcome TC.

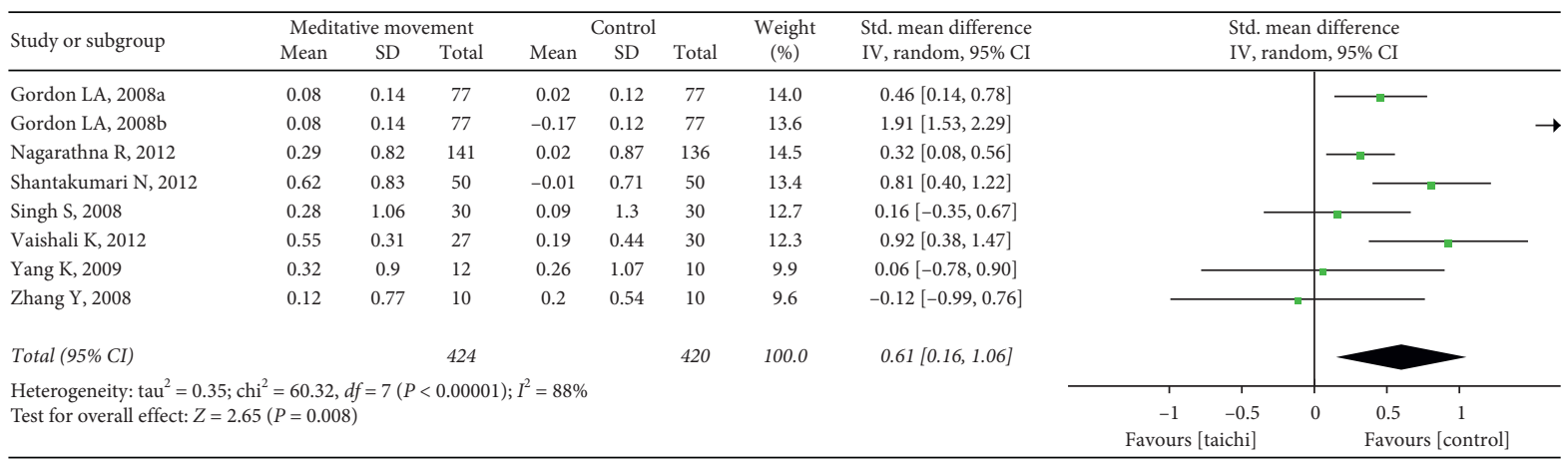

FIGURE 8: Forest plot of the comparison between meditation movements and the control group for the outcome LDL.

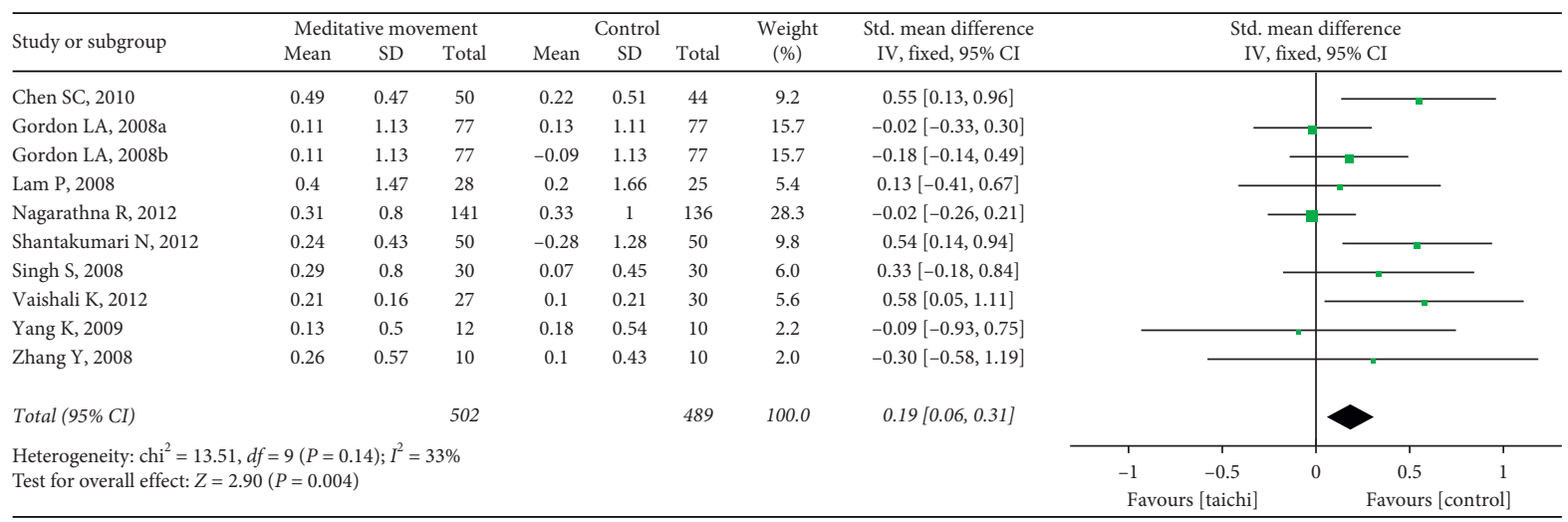

FIGURE 9: Forest plot of the comparison between meditation movements and the control group for the outcome TG. 


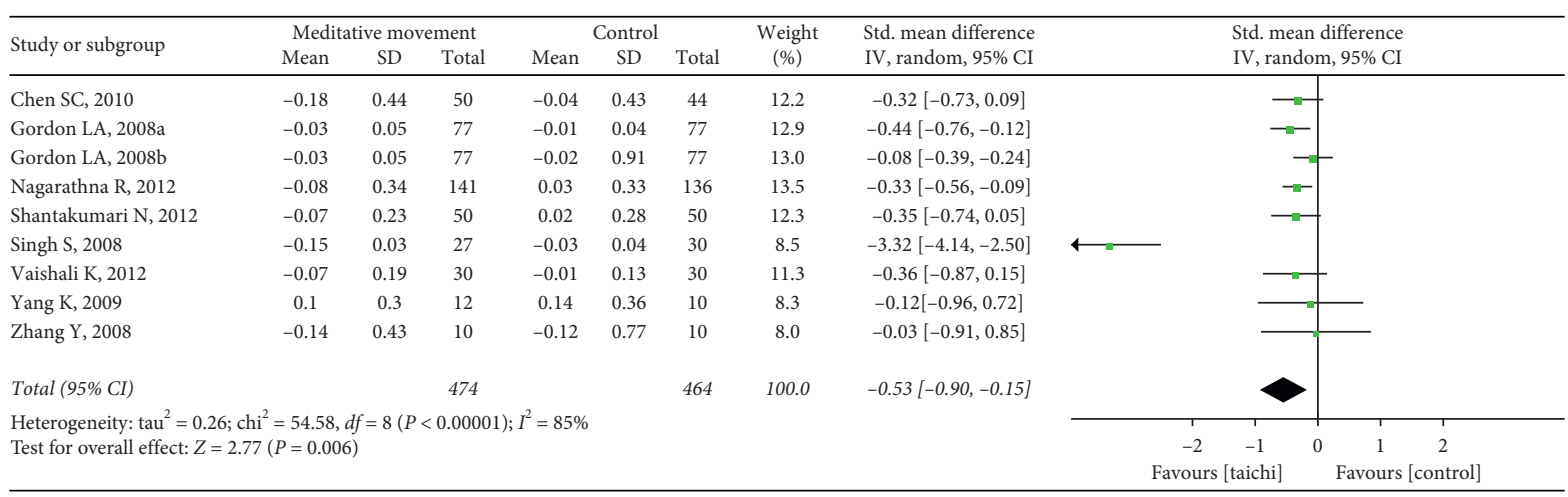

FIGURE 10: Forest plot of the comparison between meditation movements and the control group for the outcome HDL.

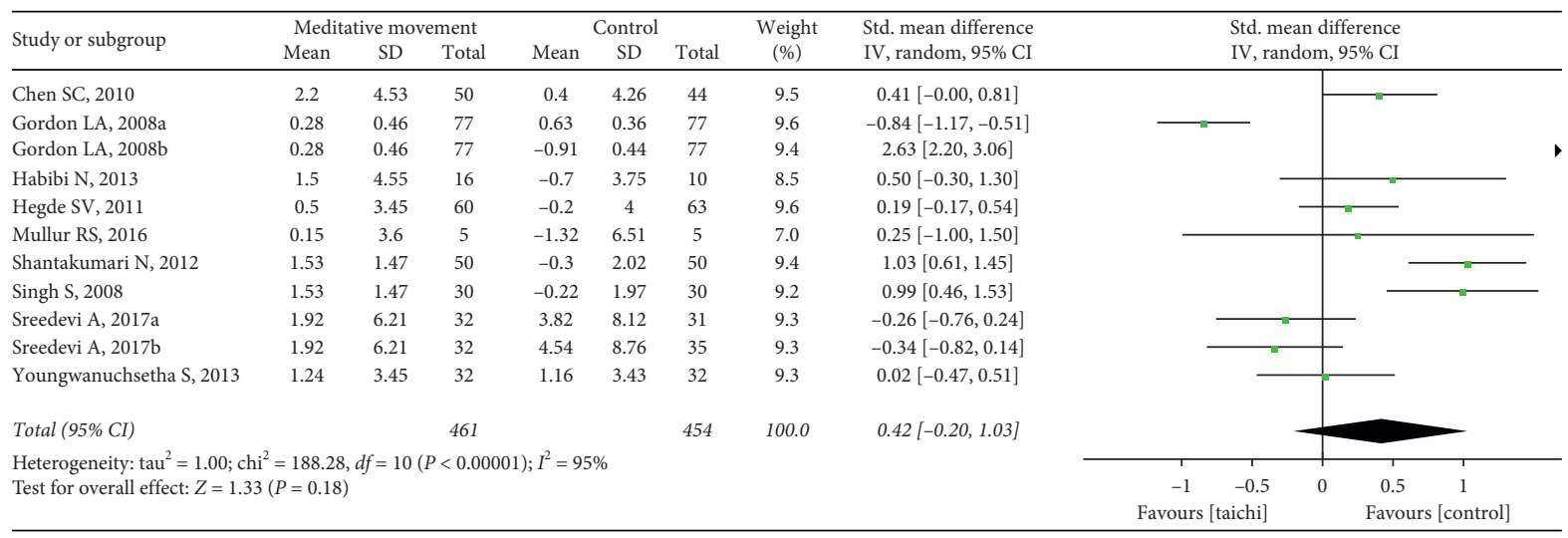

FIGURE 11: Forest plot of the comparison between meditation movements and the control group for the outcome BMI.

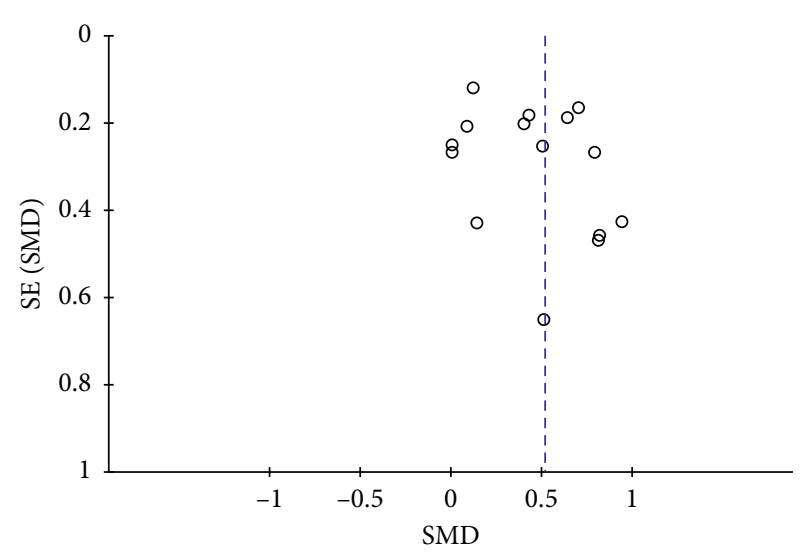

FIGURE 12: Evaluation of publication bias for FBG.

for type 2 diabetes. The particularity of meditative movements, which differs from purely physical activity, should be valued in future studies.

In summary, based on the evidence, meditative movements have significant effects on controlling blood glucose and blood lipid levels in patients with type 2 diabetes mellitus. These results support the idea that meditative movements are a possible alternative exercise for type 2 diabetes mellitus management. Due to the aforementioned limitations and potential bias, more high-quality randomized controlled studies should be conducted. In addition to increasing flexibility, muscle strength, and balance, the special effects of meditative movements in type 2 diabetes mellitus patients still need further research.

\section{Ethical Approval}

This study was based on previously published studies; therefore, ethical approval and patient consent are not relevant.

\section{Disclosure}

This paper was not commissioned and was externally peerreviewed. TWX and YY are co-first authors.

\section{Conflicts of Interest}

The authors declare that they have no conflicts of interest.

\section{Authors' Contributions}

TWX and YY contributed equally to this work.

\section{Acknowledgments}

The authors would like to thank Mr. Tao Yin for data collection. This work was funded by the National Key Research and Development Program of China (no. 2017YFC1703304), International Science and Technology 
Cooperation Project of the Department of Science and Technology of Sichuan Province (no. 18GJHZ0235), and the National Natural Science Foundation of China (no. 81873204).

\section{References}

[1] American Diabetes Association, "5. Lifestyle management: standards of medical care in diabetes-2019," Diabetes Care, vol. 42, no. 1, pp. S46-S60, 2019.

[2] D. Aune, T. Norat, M. Leitzmann, S. Tonstad, and L. J. Vatten, "Physical activity and the risk of type 2 diabetes: a systematic review and dose-response meta-analysis," European Journal of Epidemiology, vol. 30, no. 7, pp. 529-542, 2015.

[3] American Diabetes Association, "2. Classification and diagnosis of diabetes: standards of medical care in diabetes-2019," Diabetes Care, vol. 42, no. Suppl 1, pp. S13-S28, 2019.

[4] Y. Huang, IDF Diabetes Atlas, International Diabetes Federation, Belgium, China, 2017, http://www.idf.org/ouractivities/advocacy-awareness/resources-and-tools/134:idfdiabetes-atlas-8th-edition.html, 8th edition.

[5] N. H. Cho, J. E. Shaw, S. Karuranga et al., "IDF Diabetes Atlas: global estimates of diabetes prevalence for 2017 and projections for 2045," Diabetes Research and Clinical Practice, vol. 138, pp. 271-281, 2018.

[6] T. Wu, S. Qiao, C. Shi, S. Wang, and G. Ji, "Metabolomics window into diabetic complications," Journal of Diabetes Investigation, vol. 9, no. 2, pp. 244-255, 2018.

[7] R. Arturo Kobashi-Margáin, Y. Gutiérrez-Grobe, G. Ponciano-Rodríguez, M. Uribe, and N. Méndez-Sánchez, "Prevalence of type 2 diabetes mellitus and chronic liver disease: a retrospective study of the association of two increasingly common diseases in Mexico," Annals of Hepatology, vol. 9, no. 3, pp. 282-288, 2010.

[8] H. Zhao, X. Song, Z. Li et al., "Risk factors associated with nonalcohol fatty liver disease and fibrosis among patients with type 2 diabetes mellitus," Medicine (Baltimore), vol. 97, no. 37, Article ID e12356, 2018.

[9] C. D. Gardner, J. F. Trepanowski, L. C. Del Gobbo et al., "Effect of low-fat vs low-carbohydrate diet on 12-month weight loss in overweight adults and the association with genotype pattern or insulin secretion," JAMA, vol. 319, no. 7, pp. 667-679, 2018.

[10] W. M. Valencia and H. Florez, "How to prevent the microvascular complications of type 2 diabetes beyond glucose control," BMJ, vol. 356, p. i6505, 2017.

[11] American Diabetes Association, "10. Cardiovascular disease and risk management: standards of medical care in diabetes2019," Diabetes Care, vol. 42, no. Suppl 1, pp. S103-S123, 2019.

[12] L. Larkey, R. Jahnke, J. Etnier, and J. Gonzalez, "Meditative movement as a category of exercise: implications for research," Journal of Physical Activity and Health, vol. 6, no. 2, pp. 230-238, 2009.

[13] K. Diana, O. Henry, S. L. Tannenbaum et al., "Prevalence of mindfulness practices in the US workforce: national health Interview Survey," Preventing Chronic Disease, vol. 14, p. 160034, 2017.

[14] W. Lu-Ling, L. Zheng-Kun, W. Hui-Dan et al., "Effectiveness of meditative movement on COPD: a systematic review and meta-analysis," International Journal of Chronic Obstructive Pulmonary Disease, vol. 13, pp. 1239-1250, 2018.

[15] Z. Liye, Y. Albert, L. Chunxiao et al., "Effects of meditative movements on major depressive disorder: a systematic review and meta-analysis of randomized controlled trials," Journal of Clinical Medicine, vol. 7, no. 8, p. 195, 2018.

[16] F. Wang, O. Eun-Kyoung Lee, F. Feng et al., "The effect of meditative movement on sleep quality: a systematic review," Sleep Medicine Reviews, vol. 30, pp. 43-52, 2016.

[17] L. K. Larkey, S. Vega-Lopez, C. Keller et al., "A biobehavioral model of weight loss associated with meditative movement practice among breast cancer survivors," Health Psychology Open, vol. 1, no. 1, Article ID 2055102914565495, 2014.

[18] T. W. Xia, Y. Yang, W. H. Li et al., "The effect of meditative movement for glucose control in patients with type 2 diabetes: a protocol for systematic review and meta-analysis of controlled trials," Medicine (Baltimore), vol. 98, no. 19, Article ID e15639, 2019.

[19] D. Moher, A. Liberati, J. Tetzlaff, D. G. Altman, and fnm Lingua::EN::Titlecase, "Preferred reporting items for systematic reviews and meta-analyses: the PRISMA statement," BMJ, vol. 339, no. 1, p. b2535, 2009.

[20] J. J. Deeks, J. P. T. Higgins, D. G. Altman et al., Cochrane Handbook for Systematic Reviews of Interventions, The Cochrane Collaboration, London, UK, 2011.

[21] K. Yang, L. M. Bernardo, S. M. Sereika et al., "Utilization of 3month yoga program for adults at high risk for type 2 diabetes: a pilot study," Evidence-Based Complementary and Alternative Medicine, vol. 2011, Article ID 257891, 6 pages, 2011.

[22] G. S. Keerthi, P. Pal, G. K. Pal et al., "Effect of 12 Weeks of yoga therapy on quality of life and Indian diabetes risk score in normotensive Indian young adult prediabetics and diabetics: randomized control trial," Journal of Clinical \& Diagnostic Research Jcdr, vol. 11, no. 9, p. CC10, 2017.

[23] Y. Zhang and F. H. Fu, "Effects of 14-week Tai Ji Quan exercise on metabolic control in women with type 2 diabetes," The American Journal of Chinese Medicine, vol. 36, no. 4, pp. 647-654, 2008.

[24] N. Shantakumari, S. Sequeira, and R. El Deeb, "Effects of a yoga intervention on lipid profiles of diabetes patients with dyslipidemia," Indian Heart Journal, vol. 65, no. 2, pp. 127131, 2013.

[25] N. Shantakumari, S. Sequeira, and R. Eldeeb, "Effect of a yoga intervention on hypertensive diabetic patients," Journal of Advances in Internal Medicine, vol. 1, no. 2, pp. 60-63, 2012.

[26] S. Singh, T. Kyizom, K. P. Singh, O. P. Tandon, and S. V. Madhu, "Influence of pranayamas and yoga-asanas on serum insulin, blood glucose and lipid profile in type 2 diabetes," Indian Journal of Clinical Biochemistry, vol. 23, no. 4, pp. 365-368, 2008.

[27] T. Kyizom, S. Singh, K. P. Singh et al., "Effect of pranayama \& yoga-asana on cognitive brain functions in type 2 diabetes-P3 event-related evoked potential (ERP)," Indian Journal of Medical Research, vol. 131, no. 131, pp. 636-640, 2010.

[28] G.-C. Sun, J. C. Lovejoy, S. Gillham, A. Putiri, M. Sasagawa, and R. Bradley, "Effects of qigong on glucose control in type 2 diabetes: a randomized controlled pilot study," Diabetes Care, vol. 33, no. 1, p. e8, 2010.

[29] S.-C. Chen, K.-C. Ueng, S.-H. Lee, K.-T. Sun, and M.-C. Lee, "Effect of T'ai chi exercise on biochemical profiles and oxidative stress indicators in obese patients with type 2 diabetes," The Journal of Alternative and Complementary Medicine, vol. 16, no. 11, pp. 1153-1159, 2010.

[30] S. Youngwanichsetha, S. Phumdoung, and T. Ingkathawornwong, "The effects of tai chi qigong exercise on plasma glucose levels and health status of postpartum Thai women with type 2 diabetes," Focus on Alternative and Complementary Therapies, vol. 18, no. 4, pp. 182-187, 2013. 
[31] A. Sreedevi, S. K. Ramaiyer, and L. Kamalamma, "A Randomized controlled trial of the effect of yoga and peer support on glycaemic outcomes in women with type 2 diabetes mellitus: a feasibility study," BMC Complementary \& Alternative Medicine, vol. 17, no. 1, p. 100, 2017.

[32] V. Jyotsna, S. Ambekar, R. Singla et al., "Cardiac autonomic function in patients with diabetes improves with practice of comprehensive yogic breathing program," Indian Journal of Endocrinology and Metabolism, vol. 17, no. 3, pp. 480-485, 2013.

[33] S. V. Hegde, P. Adhikari, S. Kotian, V. J. Pinto, S. D'Souza, and V. D'Souza, "Effect of 3-month yoga on oxidative stress in type 2 diabetes with or without complications: a controlled clinical trial," Diabetes Care, vol. 34, no. 10, pp. 2208-2210, 2011.

[34] K. Vaishali, K. V. Kumar, P. Adhikari, and B. UnniKrishnan, "Effects of yoga-based program on glycosylated hemoglobin level serum lipid profile in community dwelling elderly subjects with chronic type 2 diabetes mellitus-a randomized controlled Trial," Physical \& Occupational Therapy In Geriatrics, vol. 30, no. 1, pp. 22-30, 2012.

[35] R. Nagarathna, M. R. Usharani, A. R. Rao, R. Chaku, R. Kulkarni, and H. R. Nagendra, "Efficacy of yoga based life style modification program on medication score and lipid profile in type 2 diabetes-a randomized control study," International Journal of Diabetes in Developing Countries, vol. 32, no. 3, pp. 122-130, 2012.

[36] R. S. Mullur and D. Ames, "Impact of a 10 minute seated yoga practice in the management of diabetes," Journal of Yoga \& Physical Therapy, vol. 6, no. 1, 2016.

[37] L. A. Gordon, E. Y. Morrison, D. A. McGrowder et al., "Effect of exercise therapy on lipid profile and oxidative stress indicators in patients with type 2 diabetes," BMC Complementary and Alternative Medicine, vol. 8, p. 21, 2008.

[38] L. Gordon, E. Y. Morrison, D. A. McGrowder et al., "Changes in clinical and metabolic parameters after exercise therapy in patients with type 2 diabetes," American Journal of Biochemistry and Biotechnology, vol. 4, no. 1, pp. 427-437, 2008.

[39] C.-M. Xiao and Y.-C. Zhuang, "Effects of Tai Chi ball on balance and physical function in older adults with type 2 diabetes mellitus," Journal of the American Geriatrics Society, vol. 63, no. 1, pp. 176-177, 2015.

[40] P. Lam, S. M. Dennis, T. H. Diamond, and N. Zwar, "Improving glycaemic and BP control in type 2 diabetes. The effectiveness of tai chi," Australian Family Physician, vol. 37, no. 10 , pp. 884-887, 2008.

[41] N. Habibi, Z. Farsani, B. Yazdani et al., "The influence of yoga on risk profiles programs in women with diabetes type II," Advances in Environmental Biology, vol. 7, pp. 550-555, 2013.

[42] T. Tsujiuchi, H. Kumano, K. Yoshiuchi et al., "The Effect of Qi-Gong relaxation exercise on the control of type 2 diabetes mellitus: a randomized controlled trial," Diabetes Care, vol. 25, no. 1, pp. 241-242, 2002.

[43] X. Liu, Y. D. Miller, N. W. Burton et al., "Qi-gong mind-body therapy and diabetes control: a randomized controlled trial," American Journal of Preventive Medicine, vol. 41, no. 2, pp. 152-158, 2011.

[44] R. Orr, T. Tsang, P. Lam, E. Comino, and M. F. Singh, "Mobility impairment in type 2 diabetes: association with muscle power and effect of tai chi intervention," Diabetes Care, vol. 29, no. 9, pp. 2120-2122, 2006.

[45] R. A. Hackett and A. Steptoe, "Type 2 diabetes mellitus and psychological stress: a modifiable risk factor," Nature Reviews Endocrinology, vol. 13, no. 9, pp. 547-560, 2017.
[46] M. Faulenbach, H. Uthoff, K. Schwegler, G. A. Spinas, C. Schmid, and P. Wiesli, "Effect of psychological stress on glucose control in patients with Type 2 diabetes," Diabetic Medicine, vol. 29, no. 1, pp. 128-131, 2011.

[47] A. Heraclides, T. Chandola, D. R. Witte, and E. J. Brunner, "Psychosocial stress at work doubles the risk of type 2 diabetes in middle-aged women: evidence from the whitehall II study," Diabetes Care, vol. 32, no. 12, pp. 2230-2235, 2009.

[48] A. M. Delamater, A. M. Jacobson, B. Anderson et al., "Psychosocial therapies in diabetes: report of the psychosocial therapies working group," Diabetes Care, vol. 24, no. 7, pp. 1286-1292, 2001.

[49] F. Zamani-Alavijeh, M. Araban, H. R. Koohestani et al., "The effectiveness of stress management training on blood glucose control in patients with type 2 diabetes," Diabetology \& Metabolic Syndrome, vol. 10, no. 1, p. 39, 2018.

[50] P. H. Black, "The inflammatory response is an integral part of the stress response: implications for atherosclerosis, insulin resistance, type II diabetes and metabolic syndrome X," Brain, Behavior, and Immunity, vol. 17, no. 5, pp. 350-364, 2003.

[51] J. D. Creswell, "Mindfulness interventions," Annual Review of Psychology, vol. 68, no. 1, p. 491, 2016.

[52] M. Xiao, Y. Zi-Qi, G. Zhu-Qing et al., "The effect of diaphragmatic breathing on attention, negative affect, and stress in healthy adults," Frontiers in Psychology, vol. 8, p. 874, 2017.

[53] T. W. Xia, Y. Yang, W. H. Li et al., "Different training durations and styles of tai chi for glucose control in patients with type 2 diabetes: a systematic review and meta-analysis of controlled trials," BMC Complementary and Alternative Medicine, vol. 19, no. 1, p. 63, 2019.

[54] J. Cui, J.-H. Yan, L.-M. Yan, L. Pan, J.-J. Le, and Y.-Z. Guo, "Effects of yoga in adults with type 2 diabetes mellitus: a metaanalysis," Journal of Diabetes Investigation, vol. 8, no. 2, pp. 201-209, 2017.

[55] D. Meng, W. Chunyan, D. Xiaosheng, and Y. Xiangren, "The effects of qigong on type 2 diabetes mellitus: a systematic review and meta-analysis," Evidence-Based Complementary and Alternative Medicine, vol. 2018, Article ID 8182938, 8 pages, 2018.

[56] X. Liu, Y. D. Miller, N. W. Burton, and W. J. Brown, “A preliminary study of the effects of Tai Chi and Qigong medical exercise on indicators of metabolic syndrome, glycaemic control, health-related quality of life, and psychological health in adults with elevated blood glucose," British Journal of Sports Medicine, vol. 44, no. 10, pp. 704-709, 2010. 